\title{
NEW COOLING SEQUENCES FOR OLD WHITE DWARFS
}

\author{
I. Renedo ${ }^{1,2}$, L. G. Althaus ${ }^{1,3}$, M. M. Miller Bertolami ${ }^{3,4}$, A. D. Romero ${ }^{3,4}$, A. H. Córsico ${ }^{3,4}$, R. D. Rohrmann ${ }^{5}$, \\ AND E. García-Berro ${ }^{1,2}$ \\ ${ }^{1}$ Departament de Física Aplicada, Universitat Politècnica de Catalunya, c/Esteve Terrades 5, 08860 Castelldefels, Spain; garcia@fa.upc.edu \\ ${ }^{2}$ Institute for Space Studies of Catalonia, c/Gran Capità 2-4. Edif. Nexus 104, 08034 Barcelona, Spain \\ ${ }^{3}$ Facultad de Ciencias Astronómicas y Geofísicas, Universidad Nacional de La Plata, Paseo del Bosque s/n, (1900) La Plata, Argentina \\ ${ }^{4}$ Instituto de Astrofísica de La Plata. IALP (CCT La Plata). CONICET-UINLP. Argentina \\ 5 Observatorio Astronómico. Universidad Nacional de Córdoba. Laprida 854, (5000) Córdoba, Argentina \\ Received 2010 March 23; accepted 2010 May 7; published 2010 June 9
}

\begin{abstract}
We present full evolutionary calculations appropriate for the study of hydrogen-rich DA white dwarfs. This is done by evolving white dwarf progenitors from the zero-age main sequence, through the core hydrogen-burning phase, the helium-burning phase, and the thermally pulsing asymptotic giant branch phase to the white dwarf stage. Complete evolutionary sequences are computed for a wide range of stellar masses and for two different metallicities, $Z=0.01$, which is representative of the solar neighborhood, and $Z=0.001$, which is appropriate for the study of old stellar systems, like globular clusters. During the white dwarf cooling stage, we self-consistently compute the phase in which nuclear reactions are still important, the diffusive evolution of the elements in the outer layers and, finally, we also take into account all the relevant energy sources in the deep interior of the white dwarf, such as the release of latent heat and the release of gravitational energy due to carbon-oxygen phase separation upon crystallization. We also provide colors and magnitudes for these sequences, based on a new set of improved non-gray white dwarf model atmospheres, which include the most up-to-date physical inputs like the Ly $\alpha$ quasi-molecular opacity. The calculations are extended down to an effective temperature of $2500 \mathrm{~K}$. Our calculations provide a homogeneous set of evolutionary cooling tracks appropriate for mass and age determinations of old DA white dwarfs and for white dwarf cosmochronology of the different Galactic populations.
\end{abstract}

Key words: stars: evolution - stars: interiors - white dwarfs

Online-only material: color figures

\section{INTRODUCTION}

White dwarfs are the most common stellar evolutionary endpoint. As a matter of fact, more than $97 \%$ of stars are expected to end their lives as white dwarfs. Their evolution can be described as a simple cooling process which lasts for very long periods of time. Moreover, since these fossil stars are abundant and long-lived objects they convey important information about the properties of all galactic populations (Hansen \& Liebert 2003). In particular, white dwarfs can be considered as reliable cosmic clocks to infer the age of a wide variety of stellar populations, such as the Galactic disk and halo (Winget et al. 1987; García-Berro et al. 1988a, 1988b, 1999; Isern et al. 1998; Torres et al. 2002), and the system of globular and open clusters (Kalirai et al. 2001; Hansen et al. 2002, 2007). They also provide helpful information about the past history of the star formation rate of our Galaxy (Díaz-Pinto et al. 1994). Additionally, they can be used to place constraints on exotic elementary particles (Isern et al. 1992, 2008; Córsico et al. 2001) or on alternative theories of gravitation (García-Berro et al. 1995; Benvenuto et al. 2004). Recent reviews on the properties and evolution of white dwarfs, and of their applications are those of Winget \& Kepler (2008) and Fontaine \& Brassard (2008). However, to use them for this wide range of applications, reliable and accurate evolutionary sequences must be computed.

Previous recent evolutionary calculations of hydrogen-rich white dwarfs are those of Fontaine et al. (2001), Salaris et al. (1997, 2000), Hansen (1998, 1999), Benvenuto \& Althaus (1999), and Wood $(1992,1995)$. All of these works have studied different aspects of the evolution of white dwarfs with hydrogen-rich envelopes. For instance, the most commonly used models, those of Wood (1995), cover a wide range of stellar masses and envelope masses and, until recently, were considered to be a standard reference in the field of white dwarf cosmochronology. However, we emphasize that these models were computed using gray atmospheres, a severe drawback (especially at low luminosities) that more recent calculations have overcome. Among these models, we mention the works of Hansen $(1998,1999)$. This set of cooling models pioneered the usage of detailed model atmospheres as surface boundary conditions in cooling calculations for old white dwarfs. This is an important issue since it affects the location of the base of the convective envelope. These calculations also showed that collision-induced absorption processes affect the colors of old white dwarfs. Salaris et al. (1997, 2000) focused on the question of interior abundance of carbon and oxygen, which is of critical importance to derive reliable ages and on the effects of phase separation of carbon and oxygen upon crystallization, while Fontaine et al. (2001) were the first to discover the importance of convective coupling between the atmosphere and the degenerate core. This last issue also bears importance for the determination of accurate ages. Nevertheless, all these works suffer from the same shortcoming. All of them evolved initial white dwarf configurations that were not obtained self-consistently from models evolving from the main sequence. As a consequence, the chemical stratification of the inner, degenerate core was simplistic in most of the cases, except in the case of the cooling sequences of Salaris et al. (1997, 2000). Also, the envelope mass and the outer layer chemical distribution were the idealized ones in all the cases. Additionally, the oldest sequences used 
physical inputs that are nowadays outdated and, finally, most of them disregarded the energy release of the carbon-oxygen phase separation (García-Berro et al. 1988a, 1988b).

The aim of this paper is to compute a set of new cooling sequences for hydrogen-rich white dwarfs, incorporating the most up-to-date physical inputs. We emphasize that our evolutionary sequences are derived from a full and self-consistent treatment of the complete evolutionary history of progenitor stars with different masses evolved with two different metallicities ( $Z=0.01$ and $Z=0.001$ ) appropriate for the study of the solar neighborhood and of metal-poor stellar systems, such as globular clusters or the galactic halo. Thus, our calculations constitute a comprehensive set of evolutionary tracks that allow us to study the evolution of hydrogen-rich white dwarfs in a wide variety of stellar systems. Moreover, since our calculations encompass the pre-white-dwarf evolutionary phases, the white dwarf evolutionary calculations presented here are not affected by inconsistencies arising from artificial procedures used to generate starting white dwarf configurations. In particular, the calculation of the evolutionary history of progenitor stars provides us with the amount of $\mathrm{H}$ left in the white dwarf and with the chemical profiles expected not only for the carbon-oxygen core, but also for the partially degenerate regions above the core, of relevance for the white dwarf cooling phase.

The paper is organized as follows. In Section 2, we describe the main physical inputs of our models. We also describe the main characteristics of the initial models, the model atmospheres employed in this work, and some details of the evolutionary computations. In Section 3, we present the results of the prewhite-dwarf evolutionary sequences, paying special attention to the derived initial-to-final mass relationship, and also the white dwarf cooling tracks. In that section, we will also discuss in detail the effects of phase separation upon crystallization. Finally, in Section 4 we summarize our results, we discuss their significance, and we draw our conclusions.

\section{COMPUTATIONAL DETAILS}

\subsection{Input Physics}

The evolutionary calculations presented in this work were done with an updated version of the LPCODE stellar evolutionary code - see Althaus et al. (2005b) and references therein. We used this code to compute both the evolution of white dwarfs and that of their progenitor stars. We emphasize that in recent years, the LPCODE stellar evolutionary code has been employed to study different aspects of the evolution of low-mass stars, such as the formation and evolution of $\mathrm{H}$-deficient white dwarfs, PG 1159, and extreme horizontal branch stars (Althaus et al. 2005b, 2009a; Miller Bertolami \& Althaus 2006; Miller Bertolami et al. 2008) and, more recently, it has also been used to study the formation of hot DQ white dwarfs (Althaus et al. 2009b), the evolution of He-core white dwarfs with high-metallicity progenitors (Althaus et al. 2009d), and the evolution of hydrogen-deficient white dwarfs (Althaus et al. 2009 c). Moreover, this code has also been used to study the white dwarf initial-final mass relationship (Salaris et al. 2009). A recent test of the code and a thorough comparison of the results obtained using LPCODE with those obtained using other evolutionary codes have recently been made in Salaris et al. (2009). Details of LPCODE can be found in these works and in Althaus et al. (2009c). For these reasons, in what follows, we only comment on the main physical input physics, namely, those that are relevant for the evolutionary calculations presented in this work.

To begin with, we recall that the radiative opacities employed in our calculations were those of the OPAL project (Iglesias \& Rogers 1996), including carbon- and oxygen-rich compositions, supplemented at low temperatures with the Alexander \& Ferguson (1994) molecular opacities. For the present calculations, we have not considered carbon-enriched molecular opacities (Marigo 2002), which are expected to reduce effective temperatures at the asymptotic giant branch (AGB; Weiss $\&$ Ferguson 2009). We adopted the conductive opacities of Cassisi et al. (2007), which cover the entire regime where electron conduction is relevant. Neutrino emission rates for pair, photo, and bremsstrahlung processes were those of Itoh et al. (1996), while for plasma processes we included the treatment presented in Haft et al. (1994).

LPCODE considers a simultaneous treatment of noninstantaneous mixing and burning of elements. Specifically, abundance changes are described by the set of equations

$$
\left(\frac{d \vec{X}}{d t}\right)=\left(\frac{\partial \vec{X}}{\partial t}\right)_{\text {nuc }}+\frac{\partial}{\partial M_{r}}\left[\left(4 \pi r^{2} \rho\right)^{2} D \frac{\partial \vec{X}}{\partial M_{r}}\right],
$$

where $\vec{X}$ is a vector containing the abundances of all the elements - see Althaus et al. (2003) for details on the numerical procedure. In this equation, the first term describes the nuclear evolution, and it is fully coupled to the current composition change due to mixing processes, as represented by the second term. In particular, the efficiency of convective mixing is described by the diffusion coefficient $D$, which in this work is given by the mixing-length theory. The nuclear network accounts explicitly for the following 16 elements: ${ }^{1} \mathrm{H},{ }^{2} \mathrm{H},{ }^{3} \mathrm{He}$. ${ }^{4} \mathrm{He},{ }^{7} \mathrm{Li},{ }^{7} \mathrm{Be},{ }^{12} \mathrm{C},{ }^{13} \mathrm{C},{ }^{14} \mathrm{~N},{ }^{15} \mathrm{~N},{ }^{16} \mathrm{O},{ }^{17} \mathrm{O},{ }^{18} \mathrm{O},{ }^{19} \mathrm{~F},{ }^{20} \mathrm{Ne}$, and ${ }^{22} \mathrm{Ne}$, together with 34 thermonuclear reaction rates of the $p p$ chains, CNO bi-cycle, helium-burning, and carbon ignition that are identical to those described in Althaus et al. (2005b), with the exception of the reaction ${ }^{12} \mathrm{C}+p \rightarrow{ }^{13} \mathrm{~N}+\gamma \rightarrow{ }^{13} \mathrm{C}+$ $e^{+}+v_{\mathrm{e}}$ and ${ }^{13} \mathrm{C}(p, \gamma){ }^{14} \mathrm{~N}$, for which we adopted the rate of Angulo et al. (1999). In particular, it is worth noting that the ${ }^{12} \mathrm{C}(\alpha, \gamma){ }^{16} \mathrm{O}$ reaction rate-which is of special relevance for the carbon-oxygen stratification of the emerging white dwarf core-adopted here is the same as that of Angulo et al. (1999). In passing, we mention that a detailed inner composition is required for a proper computation of the energy released by the redistribution of chemical elements during crystallization of the white dwarf core (see below). Specifically, the energy released during this process, and the resulting impact on the cooling ages of faint white dwarfs, increases for larger carbon abundances (Isern et al. 1997, 2000). The standard mixing-length theory for convection - with the free parameter $\alpha=1.61$ - was adopted. With this value, the present luminosity and effective temperature of the Sun, $\log T_{\text {eff }}=3.7641$ and $L_{\odot}=3.842 \times 10^{33} \mathrm{erg} \mathrm{s}^{-1}$, at an age of $4570 \mathrm{Myr}$, are reproduced by LPCODE when $Z=0.0164$ and $X=0.714$ are adopted-in agreement with the $Z / X$ value of Grevesse \& Sauval (1998).

During the evolutionary stages prior to the thermally pulsing AGB (TP-AGB) phase, we allowed the occurrence of extramixing episodes beyond each convective boundary following the prescription of Herwig et al. (1997). As well known, the occurrence of extra-mixing episodes is of relevance for the final chemical stratification of the white dwarfs, particularly during the late stage of core helium-burning phase-see Prada Moroni \& Straniero (2002) and Straniero et al. (2003). We 
treated extra-mixing as a time-dependent diffusion process-by assuming that mixing velocities decay exponentially beyond each convective boundary-with a diffusion coefficient given by $D_{\mathrm{EM}}=D_{\mathrm{O}} \exp \left(-2 z / f H_{\mathrm{p}}\right)$, where $H_{\mathrm{p}}$ is the pressure scale height at the convective boundary, $D_{\mathrm{O}}$ is the diffusion coefficient of unstable regions close to the convective boundary, and $z$ is the geometric distance from the edge of the convective boundary (Herwig et al. 1997). For all our sequences we adopted $f=0.016$, a value inferred from the width of the upper main sequence. In the case of the sequence of $1.25 M_{\odot}$ with $Z=$ 0.001 , the size of the convective core on the main sequence is small. In this case we use $f=0.008$ during the core hydrogenburning phase. Abundance changes resulting from extra-mixing were fully coupled to nuclear evolution, following Equation (1).

In the present study, extra-mixing episodes were disregarded during the TP-AGB phase. In particular, a strong reduction (a value of $f$ much smaller than 0.016 ) of extra-mixing episodes at the base of the pulse-driven convection zone seems to be supported by simulations of the $s$-process abundance patterns (Lugaro et al. 2003) and, more recently, by observational inferences of the initial-final mass relation (Salaris et al. 2009). As a result, it is expected that the mass of the hydrogen-free core of our sequences gradually grows as evolution proceeds through the TP-AGB. This is because a strong reduction or suppression of extra-mixing at the base of the pulse-driven convection zone strongly inhibits the occurrence of a third dredge-up, thus favoring the growth of the hydrogen-free core. The implications of this treatment for the theoretical initial-final mass relation will be discussed later in this paper-see also Salaris et al. (2009) and Weiss \& Ferguson (2009). The breathing pulse instability occurring toward the end of core helium-burning was suppressed-see Straniero et al. (2003) for a discussion of this point.

Mass loss was considered during core helium-burning and red giant branch phases following Schröder \& Cuntz (2005). During the AGB and TP-AGB phases, we considered the prescription of Vassiliadis \& Wood (1993). In the case of a strong reduction of third dredge-up, as occurred in our sequences, mass loss plays a major role in determining the final mass of the hydrogenfree core at the end of the TP-AGB evolution, and thus the initial-final mass relation. In particular, mass loss erodes the hydrogen-rich envelope of the star and limits the additional growth of the core mass during the TP-AGB. This is quite different from the situation in which appreciable third dredgeup takes place, in which case the final core mass at the TP-AGB phase is not very different from the mass at the first thermal pulse (Weiss \& Ferguson 2009), and the role of mass loss becomes less relevant.

We want to mention that LPCODE is able to follow the complete evolution of the star model from the main sequence through the core helium flash and, finally, for many thermal pulses on the AGB almost without manual intervention, except for the latest stages of the TP-AGB phase of the more massive sequences, where numerical instabilities arise. These instabilities, also found by other authors, are the result of the large radiation pressure in the convective envelope. To circumvent them, we found computationally convenient to artificially modify the opacity profile in those regions close to the base of the convective envelope. This procedure bears no relevance for the evolution during the white dwarf regime.

For the white dwarf regime, the physical inputs considered in LPCODE were completely revised and updated from those considered in our previous studies, particularly regarding the treatment of crystallization. With regard to the microphysics, for the high-density regime, we used the equation of state of Segretain et al. (1994), which accounts for all the important contributions for both the liquid and solid phases - see Althaus et al. (2007) and references therein. For the low-density regime, we used an updated version of the equation of state of Magni \& Mazzitelli (1979). Radiative and conductive opacities are those described at the beginning of this section. In particular, conductive opacities are considered for densities larger than that at which the main chemical constituents are completely pressure ionized. During the white dwarf regime, the metal mass fraction $Z$ in the envelope is not assumed to be fixed, rather, it is specified consistently according to the prediction of element diffusion. To account for this, we considered radiative opacities tables from OPAL for arbitrary metallicities. For effective temperatures less than $10,000 \mathrm{~K}$ we include the effects of molecular opacity by assuming pure hydrogen composition from the computations of Marigo \& Aringer (2009). This assumption is justified because element diffusion leads to pure hydrogen envelopes in cool white dwarfs. For the white dwarf regime, convection was treated in the formalism of the mixing-length theory, as given by the ML 2 parameterization (Tassoul et al. 1990).

As is well known, there are several physical processes that change the chemical abundance distribution of white dwarfs along their evolution. In particular, element diffusion strongly modifies the chemical composition profile throughout their outer layers. In this work, we computed the white dwarf evolution in a way consistent with the changes of chemical abundance distribution caused by element diffusion along the entire cooling phase. As a result, our sequences developed pure hydrogen envelopes, the thickness of which gradually increases as evolution proceeds. We considered gravitational settling and thermal and chemical diffusion - but not radiative levitation, which is only relevant at high effective temperatures for determining the surface composition-of ${ }^{1} \mathrm{H} .{ }^{3} \mathrm{He},{ }^{4} \mathrm{He},{ }^{12} \mathrm{C}$, ${ }^{13} \mathrm{C},{ }^{14} \mathrm{~N}$, and ${ }^{16} \mathrm{O}$; see Althaus et al. (2003) for details. In particular, our treatment of time-dependent diffusion is based on the multicomponent gas treatment presented in Burgers (1969). In LPCODE, diffusion becomes operative once the wind limit is reached at high effective temperatures (Unglaub \& Bues 2000 ). We assume this to occur when the surface gravity of our models $g>g_{\text {diff }}$, where $g_{\text {diff }}=7$ if $T_{\text {eff }}>90,000 \mathrm{~K}$ and $g_{\text {diff }}=6.4+T_{\text {eff }} / 150,000 \mathrm{~K}$ if $T_{\text {eff }}<90,000 \mathrm{~K}$. For smaller gravities, wind mass loss is high enough that it prevents appreciable element diffusion from occurring. This prescription represents reasonably well the detailed simulations of Unglaub $\&$ Bues (2000) for the occurrence of wind limits in hydrogenrich white dwarfs. Another physical process responsible for changes in the chemical profile of white dwarfs that we took into account is related to carbon-oxygen phase separation during crystallization. In this case, the resulting release of gravitational energy considerably impacts the white dwarf cooling times. Abundance changes resulting from residual nuclear burning - mostly during the hot stages of white dwarf evolution-and convective mixing were also taken into account in our simulations. In particular, the release of energy by proton-burning was considered down to $\log \left(L / L_{\circ}\right) \approx-4$. The role of residual hydrogen-burning in evolving white dwarfs is by no means negligible, particularly in the case of those white dwarfs resulting from low-metallicity progenitors. Finally, we considered the chemical rehomogenization of the inner carbon-oxygen profile induced by Rayleigh-Taylor instabilities following Salaris et al. (1997). These instabilities arise because 
of the positive molecular weight gradients that remain above the flat chemical profile left by convection during helium core burning.

An important aspect of the present work was the inclusion of energy sources resulting from the crystallization of the white dwarf core. This comprises the release of latent heat and the release of gravitational energy associated with changes in the carbon-oxygen profile induced by crystallization. In this study, the inclusion of these two energy sources was done selfconsistently and locally coupled to the full set of equations of stellar evolution. That is, we computed the structure and evolution of white dwarfs with the changing composition profile and with the luminosity equation appropriately modified to account for both the local contribution of energy released from the core chemical redistribution and latent heat. This constitutes an improvement over previous attempts (Salaris et al. 2000) to include the release of energy from phase separation in stellar evolutionary codes. Details about the numerical procedure to compute the energy sources from crystallization will be presented in a forthcoming work. Briefly, at each evolutionary time step we computed the crystallization temperature imposing $\Gamma=180$, where $\Gamma \equiv\left\langle Z^{5 / 3}\right\rangle e^{2} / a_{\mathrm{e}} k_{\mathrm{B}} T$ is the ion coupling constant, and $a_{\mathrm{e}}$ is the interelectronic distance, and thus taking into account the correct chemical composition of the fluid. The change of chemical composition resulting from phase separation was computed using the spindle-type phase diagram of Segretain \& Chabrier (1993). After computing the chemical compositions of both the solid and liquid phases, we evaluated the net energy released in the process as in Isern et al. (1997), and added to it the latent heat contribution, of the order of $0.77 k_{\mathrm{B}} T$ per ion, which is usually smaller. Both energy contributions were distributed over a small mass range around the crystallization front. We mention that the magnitude of both energy sources was calculated at each iteration during the convergence of the model.

\subsection{Model Atmospheres}

A proper treatment of the evolutionary behavior of cool white dwarfs requires the use of outer boundary conditions as provided by detailed non-gray model atmospheres. To this end, we considered model atmospheres that incorporate non-ideal effects in the gas equation of state and chemical equilibrium, collision-induced absorption from molecules, and the Ly $\alpha$ quasi-molecular opacity. Specifically, for $T_{\text {eff }}<10,000 \mathrm{~K}$, we derived starting values of the pressure, temperature, radial thickness, and outer mass fraction at an optical depth $\tau=25$ from a grid of non-gray model atmospheres which covers a surface gravity range between $\log g=6.5$ and 9 . For larger values of $\tau$, the use of Rosseland mean opacities is justified, and the diffusion approximation for the radiative transfer can be assumed. The use of non-gray model atmospheres to derive outer boundary conditions gives rise to shallower outer convection zones, as compared with the standard gray treatment of the atmosphere (Bergeron et al. 1997). At advanced stages of white dwarf evolution, the central temperature becomes strongly tied to the temperature stratification of the outer layers. Thus, using non-gray model atmospheres is highly desired for an accurate assessment of cooling times of cool white dwarfs (Prada Moroni \& Straniero 2007).

In the present work, model atmospheres were specifically computed on the basis of improved LTE model atmospheres. Colors and magnitudes were evaluated for effective temperatures lower than $60,000 \mathrm{~K}$, because NLTE effects become im- portant above this temperature. Calculations were done for a pure hydrogen composition and for the HST ACS filters (Vegamag system) and UBVRl photometry. The numerical code used is a new and updated version of the one described in Rohrmann et al. (2002). Models were computed assuming hydrostatic and radiative-convective equilibrium. Convective transport present in the cooler atmospheres was treated within the usual mixinglength (ML 2) approximation, adopting the same value of $\alpha$ used in the evolutionary calculations. The microphysics included in the model atmospheres comprises non-ideal effects in the gas equation of state and chemical equilibrium based on the occupation probability formalism as described in Rohrmann et al. (2002). The code includes $\mathrm{H}, \mathrm{H}_{2}, \mathrm{H}^{+}, \mathrm{H}^{-}, \mathrm{H}_{2}^{+}, \mathrm{H}_{3}^{+}, \mathrm{He}, \mathrm{He}^{-}$, $\mathrm{He}^{+}, \mathrm{He}^{2+}, \mathrm{He}_{2}^{+}, \mathrm{HeH}^{+}$, and $e^{-}$. The level occupation probabilities are self-consistently incorporated in the calculation of the line and continuum opacities. Collision-induced absorptions due to $\mathrm{H}_{2}-\mathrm{H}_{2}, \mathrm{H}_{2}-\mathrm{He}$, and $\mathrm{H}-\mathrm{He}$ pairs are also taken into account (Rohrmann et al. 2002).

For the purpose of the present work, the model atmospheres explicitly included the Ly $\alpha$ quasi-molecular opacity according to the approximation used by Kowalski \& Saumon (2006). Quasi-molecular absorption results from perturbations of hydrogen atoms by interactions with other particles, mainly $\mathrm{H}$ and $\mathrm{H}_{2}$. Here, we considered extreme pressure broadening of the line transition $\mathrm{H}(n=1) \rightarrow \mathrm{H}(n=2)$ due to $\mathrm{H}-\mathrm{H}$ and $\mathrm{H}-\mathrm{H}_{2}$ collisions, with the red wing extending far into the optical region. A detailed description of these collisional line-broadening evaluations will be presented in a forthcoming paper. On the basis of the approximations outlined in Kowalski \& Saumon (2006), we evaluated the red wing absorption within the quasistatic approach using theoretical molecular potentials to describe the interaction between the radiator and the perturber. We also considered the variation in the electric-dipole transition moment with the interparticle distance. The $\mathrm{H}_{3}$ energy-potential surfaces contributing to collisions $\mathrm{H}-\mathrm{H}_{2}$ were taken from Kulander \& Guest (1979) and Roach \& Kuntz (1986), and the dipole transition moments were calculated from Petsalakis et al. (1988). Broadening of $\mathrm{Ly} \alpha$ line by $\mathrm{H}-\mathrm{H}$ collisions plays a minor role compared to the $\mathrm{H}-\mathrm{H}_{2}$ encounters. The potential interactions for $\mathrm{H}-\mathrm{H}$ configurations were taken from Kołos \& Wolniewicz (1965) and the transition probability was assumed constant in this case. The main effect of the Ly $\alpha$ quasi-molecular opacity is a reduction of the predicted flux at wavelength smaller than $4000 \mathrm{~A}$ for white dwarfs cooler than $T_{\text {eff }} \approx 6000 \mathrm{~K}$.

\subsection{Initial Models}

The initial models for our white dwarf sequences correspond to stellar configurations derived from the full evolutionary calculations of their progenitor stars. The initial He content of our starting models at the main sequence was provided by the relation $Y=0.23+2.41 Z$, as given by present determinations of the chemical evolution of the Galaxy (Flynn 2004; Casagrande et al. 2007). Two metallicities for the progenitor stars were considered: $Z=0.01$ and 0.001 . Hence, the initial compositions of our sequences are, respectively, $(Y, Z)=(0.254,0.01)$ and $(Y, Z)=(0.232,0.001)$. All the sequences were evolved from the zero-age main sequence (ZAMS) through the thermally pulsing and mass-loss phases on the AGB and, finally, to the white dwarf cooling phase. Specifically, we computed 10 full evolutionary sequences for $Z=0.01$ and 6 for $Z=0.001$. In Table 1 , we list the initial masses of the progenitor stars at the ZAMS, together with other evolutionary quantities which will be discussed below. 
Table 1

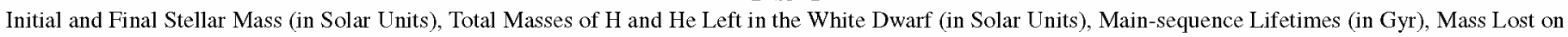
the AGB, and the Number of Thermal Pulses on the AGB for the Two Metallicities Studied

\begin{tabular}{|c|c|c|c|c|c|c|c|c|c|c|c|c|}
\hline \multirow[t]{2}{*}{$M_{\text {ZAMS }}$} & \multicolumn{6}{|c|}{$Z=0.01$} & \multicolumn{6}{|c|}{$Z=0.001$} \\
\hline & $M_{\mathrm{WD}}$ & $\log M_{\mathrm{H}}$ & $\log M_{\text {le }}$ & $t_{\mathrm{MS}}$ & $\Delta M_{\mathrm{AGB}}$ & $N_{\mathrm{TP}}$ & $M_{\mathrm{WD}}$ & $\log M_{11}$ & $\log M_{\text {!le }}$ & $t_{\mathrm{MS}}$ & $\Delta M_{\mathrm{AGB}}$ & $N_{\mathrm{TP}}$ \\
\hline 0.85 & $\ldots$ & $\ldots$ & $\ldots$ & $\ldots$ & $\ldots$ & $\pi$ & 0.505 & -3.441 & -1.567 & 11.885 & -0.016 & 2 \\
\hline 1.00 & 0.525 & -3.586 & -1.589 & 9.040 & -0.104 & 5 & 0.553 & -3.577 & -1.635 & 6.406 & -0.213 & 3 \\
\hline 1.25 & $\cdots$ & $\ldots$ & $\ldots$ & $\ldots$ & $\ldots$ & $\cdots$ & 0.593 & -3.777 & -1.840 & 2.781 & -0.499 & 7 \\
\hline 1.50 & 0.570 & -3.839 & -1.703 & 2.236 & -0.795 & 7 & 0.627 & -4.091 & -2.010 & 1.558 & -0.757 & 10 \\
\hline 1.75 & 0.593 & -3.950 & -1.851 & 1.444 & -1.081 & 10 & 0.660 & -4.174 & -1.936 & 1.049 & -1.012 & 12 \\
\hline 2.00 & 0.609 & -4.054 & -1.826 & 0.974 & -1.357 & 15 & 0.693 & -4.195 & -2.125 & 0.735 & -1.272 & 18 \\
\hline 2.25 & 0.632 & -4.143 & -1.957 & 0.713 & -1.601 & 22 & $\ldots$ & $\ldots$ & $\cdots$ & $\ldots$ & $\ldots$ & $\ldots$ \\
\hline 2.50 & 0.659 & -4.244 & -2.098 & 0.543 & -1.825 & 21 & $\ldots$ & $\ldots$ & $\cdots$ & $\ldots$ & $\ldots$ & $\ldots$ \\
\hline 3.00 & 0.705 & -4.400 & -2.270 & 0.341 & -2.279 & 19 & 0.864 & -4.860 & -2.496 & 0.279 & -2.02 & 34 \\
\hline 3.50 & 0.767 & -4.631 & -2.376 & 0.232 & -2.688 & 15 & $\cdots$ & $\cdots$ & ... & $\cdots$ & $\cdots$ & $\cdots$ \\
\hline 4.00 & 0.837 & -4.864 & -2.575 & 0.166 & -3.104 & 17 & $\ldots$ & $\ldots$ & $\ldots$ & $\ldots$ & $\ldots$ & $\ldots$ \\
\hline 5.00 & 0.878 & -4.930 & -2.644 & 0.099 & -4.029 & 12 & $\cdots$ & $\ldots$ & $\cdots$ & $\cdots$ & $\cdots$ & $\cdots$ \\
\hline
\end{tabular}

Note. The mass of the hydrogen and helium contents is given at the point of maximum effective temperature at the beginning of the white dwarf cooling branch.

\section{EVOLUTIONARY RESULTS}

\subsection{From ZAMS to the White Dwarf Stage}

The evolution in the Hertzsprung-Russell diagram of our sequences from ZAMS to advanced stages of white dwarf evolution is shown in Figure 1, for the case in which $Z=0.01$ is adopted. Note that the less massive sequence experiences a hydrogen subflash before entering its final cooling track. The initial masses at ZAMS and the final white dwarf masses of these sequences can be found in Table 1, for both metallicities. In this table, we also list the main-sequence lifetimes, which, as well known, for solar-metallicity sequences are larger than those of their metal-poor counterparts. We mention that our models have main-sequence lifetimes longer than those recently published by Weiss \& Ferguson (2009). Differences are less than $8 \%$ however, except for the main-sequence lifetime of the solar sequence, which is $25 \%$ larger. One of the reasons for such a discrepancy is due to our simplified treatment of the equation of state during the evolutionary stages prior to white dwarf formation. Also listed in this table are the total masses of the residual hydrogen and helium content left in the white dwarfs at the evolutionary stage corresponding to the point of maximum effective temperature in the Hertzsprung-Russell diagram. Note that the residual hydrogen content decreases with the white dwarf mass, a well-known result. For the case of solarmetallicity progenitors, the hydrogen mass differs by a factor of 20 for the stellar mass range being considered. This general trend is also observed for the mass of the helium content, where the mass of the residual helium ranges from 0.025 to $0.0022 M_{\circ}$. The hydrogen and helium masses listed in Table 1 should be considered as upper limits for the maximum hydrogen and helium content left in a white dwarf resulting from the evolution of single star progenitors. However, the occurrence of a late thermal pulse after departure from the TP-AGB may reduce the hydrogen mass considerably, see Althaus et al. (2005a).

Note also that for a given white dwarf mass there is a marked dependence of the final hydrogen mass on the initial metallicity of the progenitor star: higher hydrogen masses are expected in metal-poor progenitors, see Iben \& MacDonald $(1985,1986)$. For instance, for the $0.593 M_{\odot}$ white dwarf model we find that the hydrogen mass is $\log M_{\mathrm{H}} \simeq-3.950$ for the solar-metallicity progenitor, while this mass turns out to be $\log M_{\mathrm{H}} \simeq-3.777$

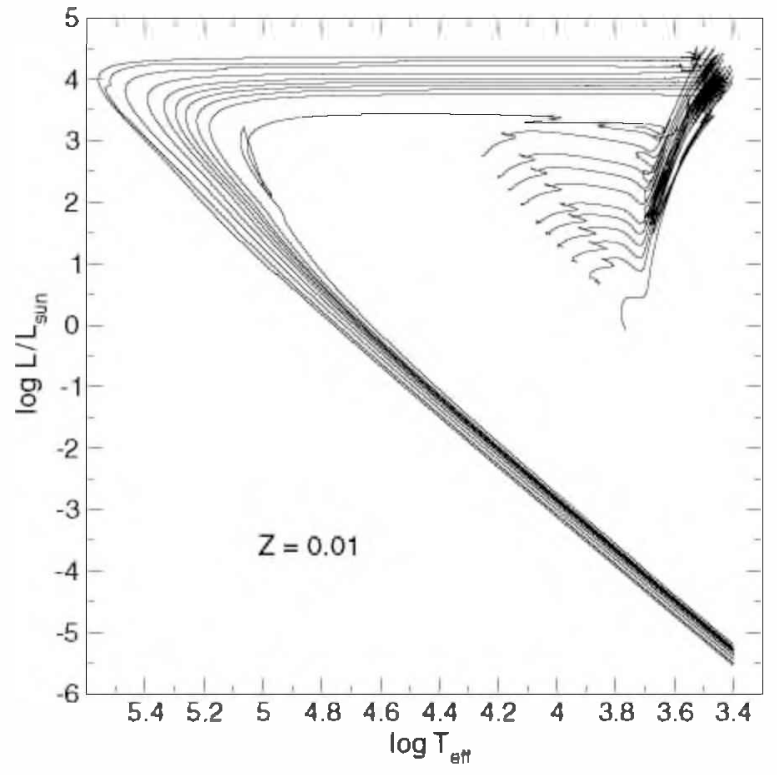

Figure 1. Hertzsprung-Russell diagram of our evolutionary sequences for $Z=0.01$. From bottom to top: evolution of the $1.0 M_{\odot}, 1.5 M_{\odot}, 1.75 M_{\odot}$, $2.0 M_{\odot}, 2.25 M_{\odot}, 2.5 M_{\odot}, 3.0 M_{\odot}, 3.5 M_{\odot}, 4.0 M_{\odot}$, and $5.0 M_{\odot}$ model stars. Evolutionary tracks are shown from ZAMS to advanced stages of white dwarf evolution. Note that the less massive sequence experiences a hydrogen subflash before entering its final cooling track.

for the metal-poor progenitor, i.e., a 50\% higher. This is an important issue since one of the factors affecting the white dwarf cooling rate is, precisely, the thickness of the hydrogenrich envelope. By contrast, in the case of the residual helium content, no appreciable dependence on the metallicity exists. Also shown in Table 1 are the number of thermal pulses during the AGB and the total mass lost during the entire AGB phase, in solar units. We find that the number of thermal pulses during the AGB phase is, generally speaking, slightly larger for the set of metal-poor evolutionary sequences. For instance, for the $1.5 M_{\odot}$ stellar sequence a total of seven thermal pulses occur for the solar-metallicity white dwarf progenitor, while for the metal-poor progenitor this number is 10 , thus leading to a more extended mass-loss phase. However, the total mass lost during the entire AGB phase is smaller for the case of a metal-poor 


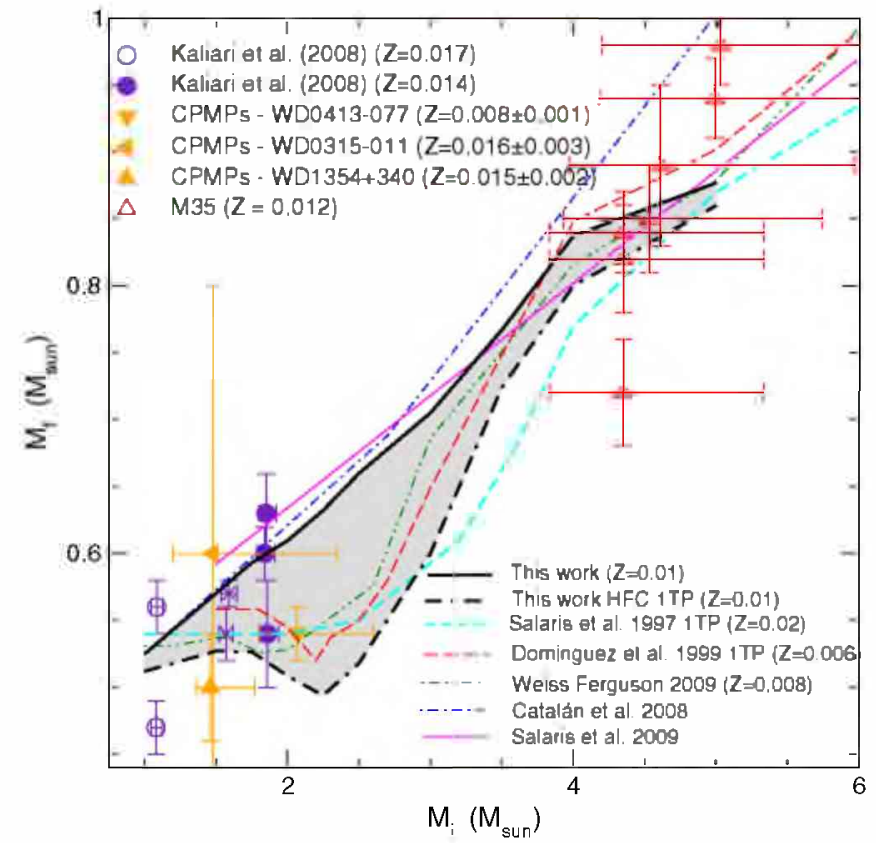

Figure 2. Theoretical initial-to-final mass relationship (thick solid line) and mass of the degenerate core at the beginning of the first thermal pulse (thick dot-dashed-dashed line) obtained in this work, both for the case in which the solar composition is adopted. The initial-to-final mass relationships of Salaris et al. (1997; short dashed line), of Domínguez et al. (1999; long dashed line), and of Weiss \& Ferguson (2009; dot-dot-dashed line) are also shown. The observational initial-to-final mass relationship of Catalán et al. (2008a) and Salaris et al. (2009) are the dot-dashed and solid lines, respectively.

(A color version of this figure is available in the online journal.)

progenitor- $0.757 M_{\odot}$ for the same model star-than for a solar-metallicity progenitor- $0.795 M_{\odot}$.

Perhaps one of the most interesting results of our full evolutionary calculations is the initial-to-final mass relationship. In Figure 2, we show our results for the case of solar composition $(Z=0.01)$. Specifically, in this figure we show using a solid line the mass of the white dwarf resulting from our theoretical calculations as a function of the initial mass at ZAMS. For the sake of comparison, we also show using a dot-dashed-dashed line the mass of the hydrogen-free core at the beginning of the first thermal pulse as a function of the initial mass of the progenitor star, and we compare this relationship with that of Salaris et al. (1997)—short dashed line-and Domínguez et al. (1999) for $Z=0.006$-long dashed line. These two initial-to-final mass relationships were obtained assuming that the mass of the resulting white dwarf corresponds to that of the hydrogen-free degenerate core at the first thermal pulse, and consequently that the core does not grow appreciably afterwards. In addition, we also show (using a dot-dot-dashed line) the recently obtained initial-to-final mass relationship of Weiss \& Ferguson (2009) for $Z=0.008$. In this calculation envelope overshooting was used during the TP-AGB, which, as mentioned in Section 2.1, considerably reduces further growth of the hydrogen-free core. We also show the observational initial-to-final mass relationship of Catalán et al. (2008b) - dot-dashed line. This relationship is based on cluster observations of different metallicities, which are close to that adopted in our calculations $(Z=0.01)$. Also shown are the observational results of Kalirai et al. (2008) — with their corresponding error bars-which correspond to metallicities very close to the solar metallicity - filled and open circles - and the results for individual white dwarfs in common proper motion pairs of Catalán et al. (2008a)—solid triangles. The mainsequence stars of these common proper motion pairs also have solar metallicity. In addition, the results for M35 as quoted in Catalán et al. (2008b), for which the estimated metallicity is also close to solar (Barrado y Navascués et al. 2001) are also shown. Finally, the semiempirical initial-to-final mass relationship of Salaris et al. (2009) based on open cluster observations is included in Figure 2.

It is to be noted that there is excellent agreement between our theoretical calculations and the empirical initial-to-final mass relationships, particularly those of Salaris et al. (2009). Also note that the mass of the hydrogen-free degenerate core at the first thermal pulse for all the theoretical sequences agree with each other and present a minimum around $\sim 2.0 M_{\odot}$, but does not agree with the empirical initial-to-final mass relationship. This emphasizes the importance of carefully following the evolution of the star models from the main sequence all the way through the TP-AGB phase and, finally, to the beginning of the white dwarf cooling track, when the mass-loss rate becomes negligible. In particular, the growth of the core mass during the TP-AGB phase is emphasized as a gray area in Figure 2. The implication of a proper computation of the initial-to-final mass relationship for the carbon/oxygen composition expected in a white dwarf will be discussed in a forthcoming paper. Also note that our pre-white-dwarf evolutionary calculations provide us with accurate and reliable starting configurations at the beginning of the white dwarf cooling phase, as they yield not only self-consistent inner chemical profiles, but also masses of the hydrogen-rich envelopes, helium buffers, and core masses which agree with the observational results.

\subsection{A Global View of the White Dwarf Cooling Phase}

In Figure 3, we show the different luminosity contributions during the white dwarf cooling phase, for an archetypical $0.609 M_{\bigcirc}$ carbon-oxygen white dwarf resulting from a progenitor star of $2.0 M_{\odot}$ with solar composition. The very first phases of the cooling phase are dominated by residual hydrogen-burning in the outer layers. This can be easily seen in Figure 3, where the different nuclear luminosities, namely, the proton-proton hydrogen-burning luminosity, the $\mathrm{CNO}$ bi-cycle luminosity, and the helium-burning luminosity are shown as a function of the cooling age. As can be seen in this figure, at high luminosities the largest contribution comes from the $\mathrm{CNO}$ bi-cycle, being the proton-proton and the helium-burning luminosities orders of magnitude smaller. This a short-lived phase (a few thousand years) and, thus, given the long-lived cooling times of white dwarfs, it is totally negligible in terms of age. Nevertheless, this phase is important as it configures the final thickness of the hydrogen-rich envelope of the white dwarf. After this short-lived phase, the nuclear luminosities abruptly decline (at $\log t \simeq 3.6$ ) and the release of gravothermal energy becomes the dominant energy source and drives the evolution. In passing, we note that residual nuclear reactions are not totally extinguished until very late phases of the evolution, in agreement with the pioneering results of Iben \& MacDonald (1985, 1986). In fact, there are still small contributions of both the $\mathrm{CNO}$ cycle and proton-proton chains until $\log t \simeq 8.3$ and $\log t \simeq 9.0$, respectively. Although almost negligible for the calculation of the cooling age in the case of white dwarfs resulting from solar-metallicity progenitors, this residual nuclear burning becomes relevant at very late stages for white dwarfs resulting from low-metallicity progenitors as it lasts for one billion years, see next section. The phase in which the evolution 


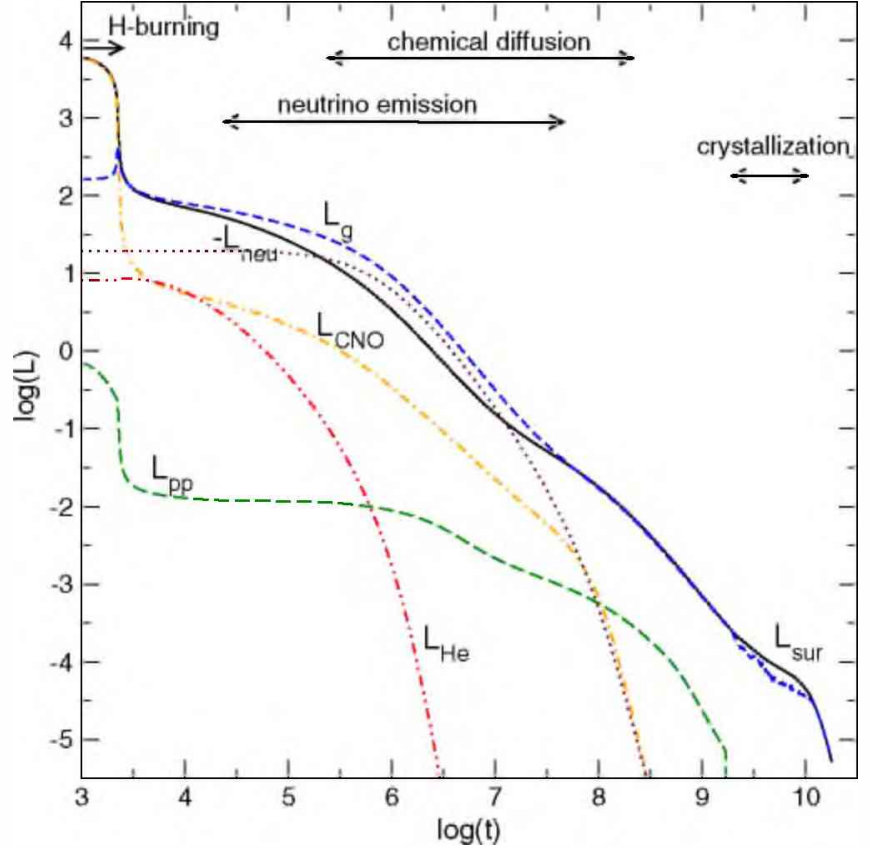

Figure 3. Time dependence of the different luminosity contributions for our $0.609 M_{\odot}$ white dwarf sequence when carbon-oxygen phase separation is included. We show the photon luminosity, $L_{\text {sur }}$ (solid line), the luminosities due to nuclear reactions-proton-proton chains, $L_{p p}$ (long dashed line), CNO bi-cycle, $L_{\mathrm{CNO}}$ (dot-dashed line), helium-burning, $L_{\mathrm{He}}$ (dot-dot-dashed line)-the neutrino losses, $L_{\text {neu }}$ (dotted line) and the rate of gravothermal (compression plus thermal) energy release, $L_{\mathrm{g}}$ (dashed line). Time is expressed in years counted from the moment at which the remnant reaches $\log T_{\text {eff }}=4.87$ at high luminosity. The various physical processes occurring as the white dwarf cools down are indicated in the figure. The progenitor corresponds to a solarmetallicity $2.0 M_{\odot}$ star.

(A color version of this figure is available in the online journal.)

is driven by gravitational contraction lasts for about one million years. During this phase, the release of gravothermal energy occurs preferentially in the outer partially or non-degenerate layers of the white dwarf. More or less at the same epoch-that is, at $\log (t) \simeq 5.6$-neutrino losses also become important. In particular, at this epoch neutrinos are the dominant energy sink in the degenerate core of the white dwarf, and their associated luminosity becomes larger than the optical luminosity. In fact, during a relatively long period of time (from $\log t \sim 5.6$ to 7.1) the neutrino luminosity is comparable to the luminosity associated with the gravothermal energy release. It is also interesting to note that at approximately the same time, element diffusion is operating in the outer partially degenerate envelope, shaping the chemical stratification of the very outer layers of the white dwarf. We will discuss below the resulting chemical stratification. This phase lasts for about $2.2 \times 10^{8} \mathrm{yr}$. At $\log t \sim 7.8$ the temperatures in the degenerate core decrease below the threshold where neutrino emission ceases and, consequently, the neutrino luminosity abruptly drops. During this phase of the evolution most of the energy released by the white dwarf has gravothermal origin, and the white dwarf cools according to the classical Mestel's law (Mestel 1952). Finally, at $\log t \simeq 9.2$ crystallization sets in at the center of the white dwarf and the cooling process slows down due to the release of latent heat and of gravitational energy due to carbon-oxygen phase separation. These physical processes are noticeable as a change in the slope of the cooling curve. Also note that during the crystallization phase the surface luminosity is larger than the gravothermal luminosity, a consequence of these two addi-

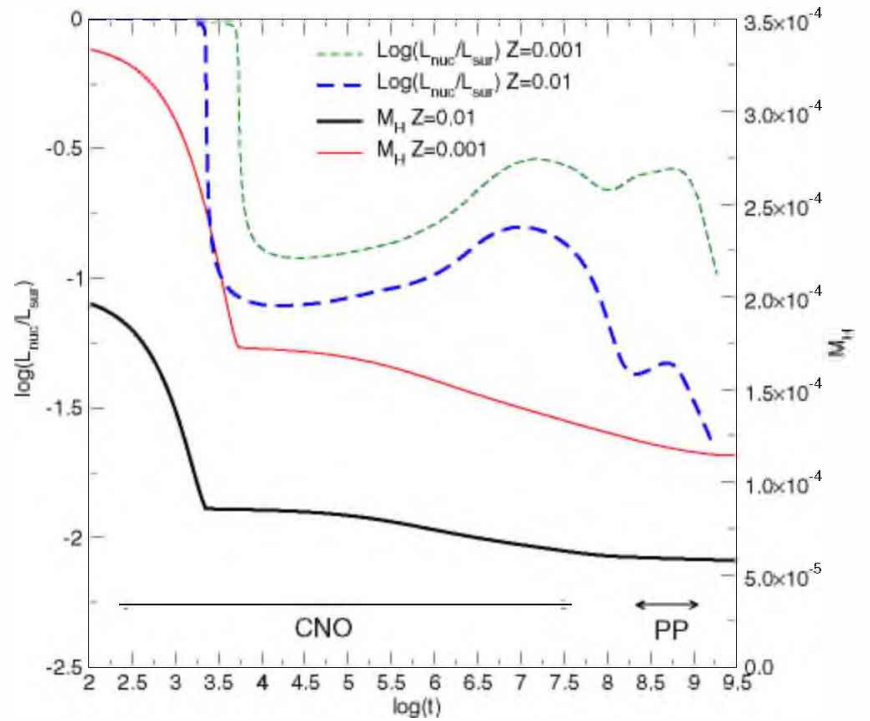

Figure 4. Temporal evolution of the hydrogen content $M_{\mathrm{H}}$ (in solar masses) and the ratio of hydrogen (proton-proton chains and $\mathrm{CNO}$ bi-cycle) nuclear burning to surface luminosity, solid and dashed lines, respectively. Thick (thin) lines correspond to progenitors with $Z=0.01(Z=0.001)$. Note that the hydrogen content left in the white dwarf, and therefore the nuclear energy output, is strongly dependent on the metal content of the progenitor stars. Although a large fraction of the hydrogen content is burnt before the remnant reaches the terminal cooling track at young ages, note that in the case of low metallicity, residual burning during the white dwarf stage reduces the hydrogen content considerably. The mass of the white dwarf corresponding to $Z=0.01$ $(Z=0.001)$ is $0.609 M_{\odot}\left(0.593 M_{\odot}\right)$.

(A color version of this figure is available in the online journal.)

tional energy sources. This phase lasts for $\sim 9.4 \times 10^{9} \mathrm{yr}$. After this phase, the temperature of the crystallized core of the white dwarf drops below the Debye temperature and the heat capacity of the white dwarf drops. Consequently, the white dwarfs enter the so-called Debye cooling phase, and the slope of the cooling curve increases again. This occurs at $\log t \sim 10$.

\subsection{The Thickness of the Hydrogen Envelope}

Figure 4 shows the temporal evolution of the masses of the hydrogen content for two representative white dwarf cooling tracks of the two metallicities explored here. Also shown are the ratio of the hydrogen-burning luminosities to the total luminosity. In particular, the thick lines represent the evolution of a $0.609 M_{\circ}$ white dwarf resulting from a solar progenitor, while the thin lines show that of a $0.593 M_{\odot}$ white dwarf resulting from a metal-poor progenitor. The solid lines correspond to the evolution of the mass of the hydrogen content, while the dashed lines show the evolution of the nuclear luminosities. As can be seen, residual hydrogen-burning is dominant during the first evolutionary phases of the white dwarf stage. As a consequence, the mass of these envelopes decreases for a period of time of $\sim 3 \times 10^{3} \mathrm{yr}$, during which hydrogen-burning supplies most of the surface luminosity of the white dwarf. However, as soon as the mass of the hydrogen content decreases below a certain threshold $\left(\sim 8 \times 10^{-5} M_{\odot}\right.$ for the solar-metallicity star and $\sim 1.7 \times 10^{-4} M_{\odot}$ for the metal-poor star) the pressure at the bottom of the envelope is not large enough to support further nuclear reactions, and hence the main energy source of the white dwarf is no longer nuclear reactions but gravothermal energy release, and the hydrogen content reaches a stationary value. This is true in the case of the white dwarf with a solar-metallicity progenitor, but for the white dwarf remnant that results from 


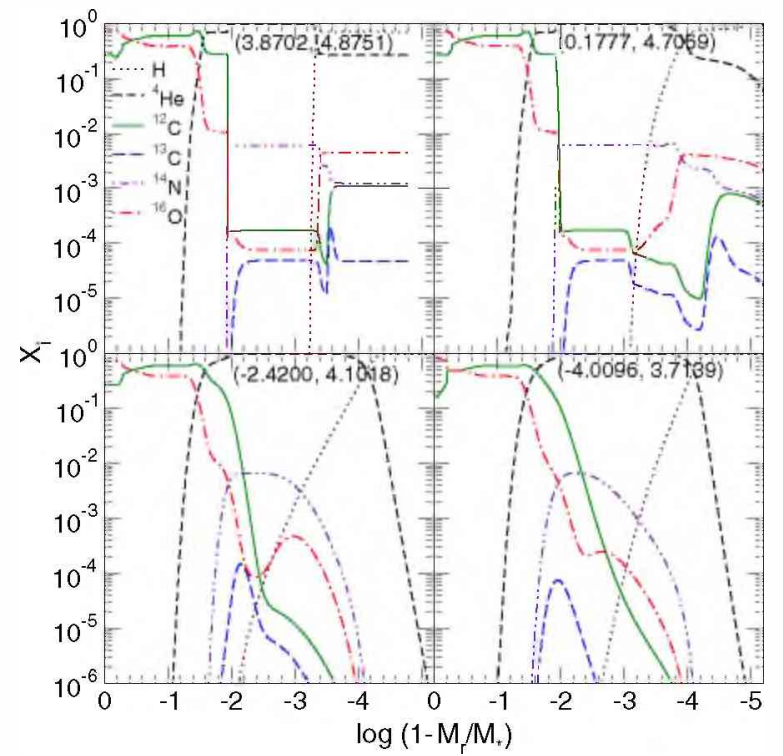

Figure 5. Abundance by mass of $\mathrm{H},{ }^{4} \mathrm{He},{ }^{12} \mathrm{C},{ }^{13} \mathrm{C},{ }^{14} \mathrm{~N}$, and ${ }^{16} \mathrm{O}$ as a function of the outer mass fraction $\log \left(1-M_{r} / M_{*}\right)$ for our $0.609 M_{\mathcal{C}^{*}}$ white dwarf sequence at various selected evolutionary stages. The upper left panel corresponds to the start of the cooling branch ( $\log T_{\mathrm{eff}}=4.87$ at high luminosity). The upper right panel shows the chemical profiles after some diffusion has already taken place in the envelope. The bottom left panel shows the situation at the domain of the pulsating DA white dwarfs. Finally, the bottom right panel shows the chemical abundances after the onset of crystallization. Luminosity and effective temperatures $\left(\log L / L_{\bigcirc}, \log T_{\text {eff }}\right)$ are specified for each stage. The metallicity of the progenitor star is $Z=0.01$.

(A color version of this figure is available in the online journal.)

the lower metallicity progenitor star, it is clear that residual hydrogen-burning is by no means negligible. In this case, note that hydrogen-burning represents an important fraction of the surface luminosity after $\approx 10^{7} \mathrm{yr}$ of evolution, and even at more advanced stages $\left(\approx 10^{9} \mathrm{yr}\right)$, this contribution reaches up to $30 \%$. At this time, the nuclear energy production is almost entirely from the proton-proton chain, and the hydrogen content has been reduced down to $\sim 1.1 \times 10^{-4} M_{\odot}$. The contribution of hydrogen-burning to surface luminosity increases for white dwarfs with lower stellar masses. We would like to emphasize at this point the importance of computing self-consistently the very first stages of the white dwarf evolution, as they provide a homogeneous set of white dwarf envelope masses, which as the evolution proceeds influence the cooling of white dwarfs.

\subsection{The Chemical Abundances of the Envelope}

Figure 5 shows the chemical profiles of the $0.609 M_{\odot}$ white dwarf resulting from a solar-metallicity progenitor for selected evolutionary stages along the white dwarf cooling track. Each of the panels is labeled with the luminosity and effective temperature of the evolutionary stage. In these panels, we show the abundance profiles of hydrogen, helium, carbon, nitrogen, and oxygen in terms of the outer mass fraction. As can be seen in the upper left panel, which depicts the chemical profiles at the beginning of the cooling track, the resulting white dwarf has a hydrogen-rich envelope, with substantial amounts of heavier elements, such as helium, carbon, nitrogen, and oxygen. The chemical composition of this layer is similar to that of typical AGB stars that have not experienced third dredge-up episodes, with oxygen being more abundant than carbon, and nitrogen almost as abundant as carbon. Specifically, these abundances are essentially fixed by the first dredge-up episode during the red giant phase. The deeper layers in the helium buffer zone show CNO abundances that reflect the occurrence of hydrogenburning in prior stages, with nitrogen far more abundant than carbon and oxygen. As the white dwarf evolves across the knee in the Hertzsprung-Russell diagram, gravitational settling and diffusion become the relevant physical processes and the heavier chemical elements begin to sink appreciably. This is illustrated in the upper right panel of Figure 5. As can be seen in this panel, at this stage, the white dwarf has already developed a thin pure hydrogen envelope that thickens as evolution proceeds. Note that at this evolutionary stage some diffusion and gravitational settling has already occurred in deeper layers, and the chemical interfaces exhibit less sharp discontinuities. During these stages, the chemical composition has also changed as a result of nuclear burning via the $\mathrm{CN}$ cycle at the base of the hydrogen envelope. With further cooling - see the bottom left panel of Figure 5-the action of element diffusion becomes more apparent. In fact, the helium-rich buffer increases its size and both carbon and oxygen sink toward deeper and deeper regions of the white dwarf. Also, the thickness of the hydrogen-rich layer increases appreciably, and at the same time, the tail of the hydrogen distribution continues to chemically diffuse inward. At this stage, which corresponds to the domain of the pulsating DA white dwarfs, a rather thick hydrogen envelope has been formed, and below it, a helium-rich and several very thin layers, which are rich in even heavier elements - a consequence of the high gravity of the white dwarf. Finally, the bottom right panel depicts the situation after the onset of crystallization. Note the change in the carbon and oxygen composition of the core as a result of crystallization. This sequence of figures emphasizes the importance of a proper treatment of time-dependent diffusion processes during white dwarf evolution, and the extent to which the initial chemical stratification at the start of the cooling phase is altered by these processes.

\subsection{Convective Coupling and Crystallization}

As discussed in Section 3.2, the cooling curve is influenced strongly by crystallization. However, at this evolutionary stage the slope of the cooling curve is not only dictated by the release of latent heat and other energy sources associated with crystallization but also by the so-called convective coupling. When the envelope becomes fully convective the inner edge of the convective region reaches the boundary of the degenerate regions (Fontaine et al. 2001). This effect is illustrated in Figure 6, where we show as a function of the surface luminosity (in solar units), the evolution of the cooling times and central temperatures (left scales), and the mass of the crystallized white dwarf core (right scale) for two white dwarfs, a low-mass white dwarf of $0.525 M_{\odot}$ and a rather massive white dwarf with $M=0.878 M_{\odot}$, both resulting from solar-metallicity progenitors. As can be seen, as both the white dwarfs cool, there is a gradual decrease of the central temperatures, while their corresponding cooling times also smoothly increase. At approximately $\log \left(L / L_{\odot}\right) \simeq-3.8$ crystallization sets in for the less massive white dwarf, whereas for the massive star this occurs at $\log \left(L / L_{\bigcirc}\right) \simeq-3$. Convective coupling between the degenerate core and the partially degenerate convective envelope also occurs at low luminosities. Since the inner edge of the convective envelope reaches the boundary of the core, an increase in the rate of energy transfer across the outer opaque envelope occurs, which is much more efficient than radiative transfer alone. As a consequence, the relation between the central temperature and the surface luminosity experiences a 
Table 2

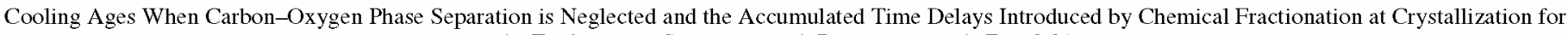
the Evolutionary Sequences with Progenitors with $Z=0.01$

\begin{tabular}{|c|c|c|c|c|c|c|c|c|c|c|}
\hline \multirow[t]{2}{*}{$-\log \left(L / L_{\odot}\right)$} & \multicolumn{10}{|c|}{$t(\mathrm{Gyr})$} \\
\hline & $0.525\left(M_{\odot}\right)$ & $0.570\left(M_{(\mathrm{B}}\right)$ & $0.593\left(M_{(}\right)$ & $0.609\left(M_{\circlearrowleft}\right)$ & $0.632\left(M_{\odot}\right)$ & $0.659\left(M_{\odot}\right)$ & $0.705\left(M_{\subseteq 1}\right)$ & $0.767\left(M_{\odot}\right)$ & $0.837\left(M_{\circlearrowleft}\right)$ & $0.878\left(M_{\circlearrowleft}\right)$ \\
\hline 2.0 & 0.17 & 0.17 & 0.17 & 0.17 & 0.17 & 0.17 & 0.18 & 0.20 & 0.21 & 0.22 \\
\hline 3.0 & 0.80 & 0.80 & 0.80 & 0.80 & 0.82 & 0.85 & 0.89 & 0.95 & 1.03 & 1.07 \\
\hline 4.0 & 3.97 & 4.17 & 4.26 & 4.25 & 4.26 & 4.34 & 4.57 & 4.75 & 4.89 & 4.92 \\
\hline 4.2 & 6.57 & 6.79 & 6.85 & 6.85 & 6.86 & 6.95 & 7.10 & 7.02 & 6.80 & 6.62 \\
\hline 4.4 & 8.88 & 9.25 & 9.32 & 9.34 & 9.38 & 9.52 & 9.73 & 9.66 & 9.42 & 9.17 \\
\hline 4.6 & 10.69 & 11.03 & 11.13 & 11.19 & 11.25 & 11.40 & 11.65 & 11.59 & 11.36 & 11.08 \\
\hline 4.8 & 12.40 & 12.71 & 12.77 & 12.81 & 12.84 & 12.96 & 13.18 & 13.04 & 12.71 & 12.39 \\
\hline \multirow[t]{2}{*}{5.0} & 14.13 & 14.33 & 14.32 & 14.34 & 14.30 & 14.37 & 14.50 & 14.22 & 13.74 & 13.32 \\
\hline & \multicolumn{10}{|c|}{$\delta t$ (Gyr) } \\
\hline 3.0 & 0.00 & 0.00 & 0.00 & 0.00 & 0.00 & 0.00 & 0.00 & 0.00 & 0.00 & 0.01 \\
\hline 4.0 & 0.10 & 0.15 & 0.16 & 0.23 & 0.27 & 0.37 & 0.67 & 0.97 & 1.14 & 1.20 \\
\hline 4.2 & 0.64 & 0.87 & 0.88 & 0.97 & 1.00 & 1.12 & 1.39 & 1.47 & 1.48 & 1.49 \\
\hline 4.4 & 1.03 & 1.26 & 1.28 & 1.30 & 1.33 & 1.45 & 1.66 & 1.72 & 1.68 & 1.67 \\
\hline 4.6 & 1.15 & 1.33 & 1.37 & 1.38 & 1.40 & 1.51 & 1.72 & 1.76 & 1.72 & 1.72 \\
\hline
\end{tabular}

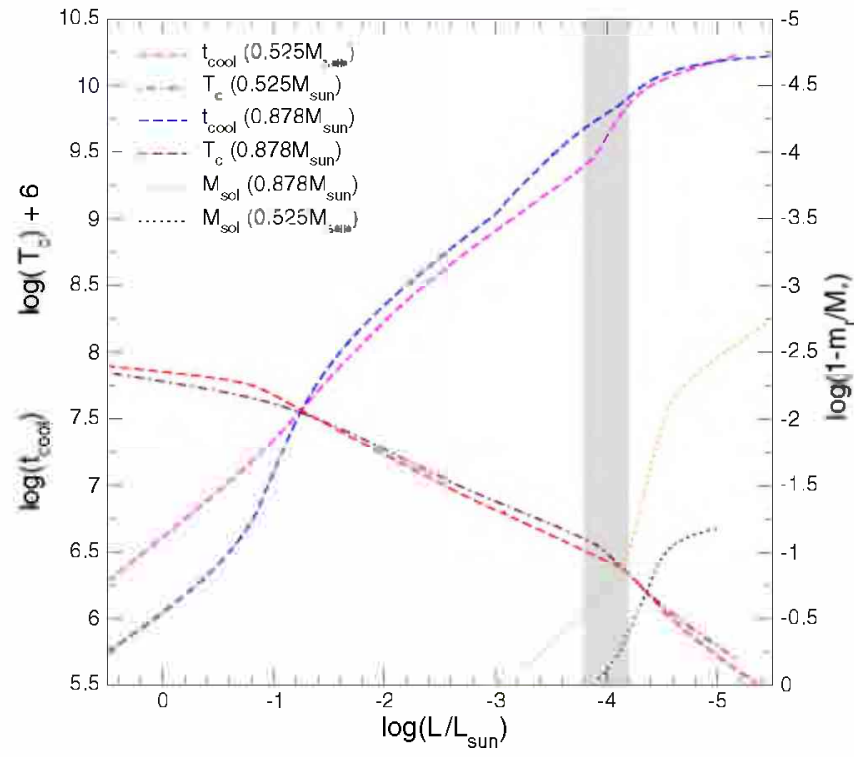

Figure 6. Some evolutionary properties corresponding to our $0.878 M_{\odot}$ and $0.525 M_{\odot}$ white dwarf sequences with carbon-oxygen phase separation resulting from $Z=0.01$ progenitors. We show in terms of the surface luminosity the run of the cooling times (dashed lines), of the central temperature (dot-dashed lines), both read on the left-hand side scale, and the evolution of the growth of the crystallized core (dotted lines) as given by the outer mass fraction on the right-hand side scale. The gray area marks the occurrence of convective coupling. The energy released due to convective coupling and the energy resulting from the crystallization process markedly impact the cooling curves, see the text for details.

(A color version of this figure is available in the online journal.)

sudden change of slope, which can be clearly seen in Figure 6, where we show the region in which convective coupling occurs as a shaded area. Note that in the case of the more massive white dwarf, convective coupling takes place at luminosities markedly lower than that at which crystallization starts in the core. In fact, more than $90 \%$ of the white dwarf mass has crystallized by the time convective coupling occurs in the $0.878 M_{\odot}$ white dwarf. In contrast, for the less massive white dwarf, both crystallization and convective coupling occur at approximately the same stellar luminosity, and thus the resulting impact of these effects on the rate of cooling is more noticeable in this case.

\subsection{Cooling Times and Chemical Composition of the Core}

One of the most noticeable features of the white dwarf cooling tracks presented here is the inclusion in a self-consistent manner of the release of gravitational energy due to phase separation of carbon and oxygen upon crystallization. Previous studies of this kind (Salaris et al. 2000) included the effects of phase separation, but a semianalytical approach was used. To highlight the importance of phase separation upon crystallization, we have computed two different sets of cooling sequences. In the first of these cooling sequences phase separation of carbon and oxygen was fully taken into account, whereas in the second it was disregarded. In Table 2, we list for various luminosities the cooling ages of all our white dwarf sequences resulting from solar-metallicity progenitors, when carbon-oxygen phase separation is neglected (top section), and the corresponding delays introduced by carbon-oxygen phase separation. We also show the same quantities for the case of metal-poor progenitors in Table 3. Clearly, phase separation of carbon and oxygen introduces significant delays at low luminosities, between 1.0 and $1.8 \mathrm{Gyr}$. It is worth mentioning that at $\log \left(L / L_{\circ}\right)=-4.6$, a luminosity slightly smaller than that of the observed drop-off in the disk white dwarf luminosity function, $\log \left(L / L_{\odot}\right) \simeq-4.5$, phase separation of carbon and oxygen represents a correction of $\sim 15 \%$ to the total age that although not very large, is not negligible either, if precise cosmochronology is to be performed. At $\log \left(L / L_{\odot}\right)=-4.0$, the delays constitute $\sim 25 \%$ of the age for the more massive white dwarfs. Note that the magnitude of the delays increases with the mass of the white dwarf. For the case of white dwarfs resulting from metal-poor progenitors (see Table 3), and for the same fiducial luminosity the delays introduced by carbon-oxygen phase separation are slightly larger for the same white dwarf mass. Our computed delays are larger than those obtained by Salaris et al. (2000). For instance, for our $0.609 M_{\circ}$ white dwarf cooling sequence at $\log \left(L / L_{\circ}\right)=-4.6$ we obtain $\delta t \simeq 1.38 \mathrm{Gyr}$, while Salaris et al. (2000) at the same luminosity obtain for their $0.61 M_{\odot}$ white dwarf evolutionary sequence $\delta t \simeq 1.00 \mathrm{Gyr}$. This difference stems in part from the larger carbon abundances of our white dwarf model, which leads to a larger energy release of the carbon-oxygen phase separation process, and consequently to larger time delays. Indeed, the chemical profiles used by Salaris et al. (2000) were those 
Table 3

Same as Table 2 but for $Z=0.001$

\begin{tabular}{|c|c|c|c|c|c|c|c|}
\hline \multirow[t]{2}{*}{$-\log \left(L / L_{0}\right)$} & \multicolumn{7}{|c|}{$t$ (Gyr) } \\
\hline & $\underline{0.505\left(M_{\odot}\right)}$ & $0.553\left(M_{\odot}\right)$ & $0.593\left(M_{\odot}\right)$ & $0.627\left(M_{\odot}\right)$ & $0.660\left(M_{\odot}\right)$ & $0.693\left(M_{\odot}\right)$ & $0.864\left(M_{\odot}\right)$ \\
\hline 2.0 & 0.24 & 0.22 & 0.19 & 0.17 & 0.18 & 0.19 & 0.22 \\
\hline 3.0 & 1.01 & 1.03 & 0.91 & 0.83 & 0.86 & 0.89 & 1.06 \\
\hline 4.0 & 4.15 & 4.49 & 4.50 & 4.40 & 4.48 & 4.59 & 4.98 \\
\hline 4.2 & 6.74 & 7.15 & 7.11 & 7.01 & 7.13 & 7.21 & 6.84 \\
\hline 4.4 & 9.01 & 9.57 & 9.61 & 9.54 & 9.73 & 9.84 & 9.42 \\
\hline 4.6 & 10.81 & 11.39 & 11.43 & 11.43 & 11.63 & 11.74 & 11.37 \\
\hline 4.8 & 12.56 & 13.10 & 13.08 & 13.04 & 13.22 & 13.30 & 12.67 \\
\hline \multirow[t]{2}{*}{5.0} & 14.33 & 14.78 & 14.64 & 14.53 & 14.66 & 14.64 & 13.66 \\
\hline & \multicolumn{7}{|c|}{$\delta t$ (Gyr) } \\
\hline 3.0 & 0.00 & 0.00 & 0.00 & 0.00 & 0.00 & 0.00 & 0.00 \\
\hline 4.0 & 0.08 & 0.17 & 0.16 & 0.25 & 0.39 & 0.49 & 1.14 \\
\hline 4.2 & 0.49 & 0.80 & 0.94 & 1.14 & 1.24 & 1.27 & 1.44 \\
\hline 4.4 & 0.90 & 1.18 & 1.34 & 1.49 & 1.55 & 1.57 & 1.63 \\
\hline 4.6 & 1.04 & 1.28 & 1.42 & 1.58 & 1.61 & 1.64 & 1.70 \\
\hline
\end{tabular}

of Salaris et al. (1997). The central carbon abundance for the $0.61 M_{\odot}$ white dwarf is $X_{\mathrm{C}} \simeq 0.25$, while for our $0.609 M_{\odot}$ model we obtain $X_{\mathrm{C}} \simeq 0.29$. Hence, the delays introduced by carbon-oxygen phase separation are correspondingly larger in our model. A realistic core composition is crucial for a proper assessment of the energy release from phase separation and its impact on the cooling times.

Figure 7 shows the evolutionary cooling sequences of several selected white dwarfs. Specifically, we show the luminosity as a function of the cooling age of white dwarfs with solarmetallicity progenitors and masses $0.525 M_{\odot}$ (upper left panel), $0.570 M_{\odot}$ (upper right panel), $0.609 M_{\odot}$ (bottom left panel), and $0.877 M_{\odot}$ (bottom right panel), respectively. The figure emphasizes the evolutionary stages where the processes of convective coupling, crystallization, and Debye cooling take place. As mentioned above, in low-mass white dwarfs, crystallization and convective coupling occur approximately at the same luminosity, $\log \left(L / L_{\odot}\right) \approx-4$, thus resulting in a pronounced impact on the cooling rate. As can be observed, the cooling tracks presented here have been computed down to very low luminosities, typically of the order of $10^{-5} L_{\odot}$, or even smaller. At these very low luminosities the central regions of the white dwarf have low enough temperatures to enter the so-called Debye cooling phase. In this phase, the specific heat drops abruptly as $T^{3}$, and as a consequence the cooling rate is enhanced. Thus, the cooling curve rapidly drops. The transition to this phase of cooling depends on the Debye temperature, $\theta_{\mathrm{D}}$, which in turn depends on the density $\left(\theta_{\mathrm{D}} \propto \rho^{1 / 2}\right)$. Consequently, more massive white dwarfs enter this phase at larger temperatures (luminosities). In our most massive sequence, rapid Debye cooling is expected to occur at the lowest luminosities we computed, as it is clear from Figure 7, while for lower stellar masses, this phase is delayed to much lower luminosities.

Phase separation in the deep interiors of white dwarfs also has obvious imprints in the chemical profiles of carbon and oxygen. To illustrate this in Figure 8 we display for four selected white dwarf evolutionary sequences the oxygen mass abundance as a function of the interior mass at three selected evolutionary stages. Specifically, we show the abundance profiles shortly after the progenitor star departs from the AGB (dashed lines), the same profiles after Rayleigh-Taylor rehomogeneization (Salaris et al. 1997) have occurred (solid lines), and finally, when the entire white dwarf core has crystallized (dot-dot-dashed lines).

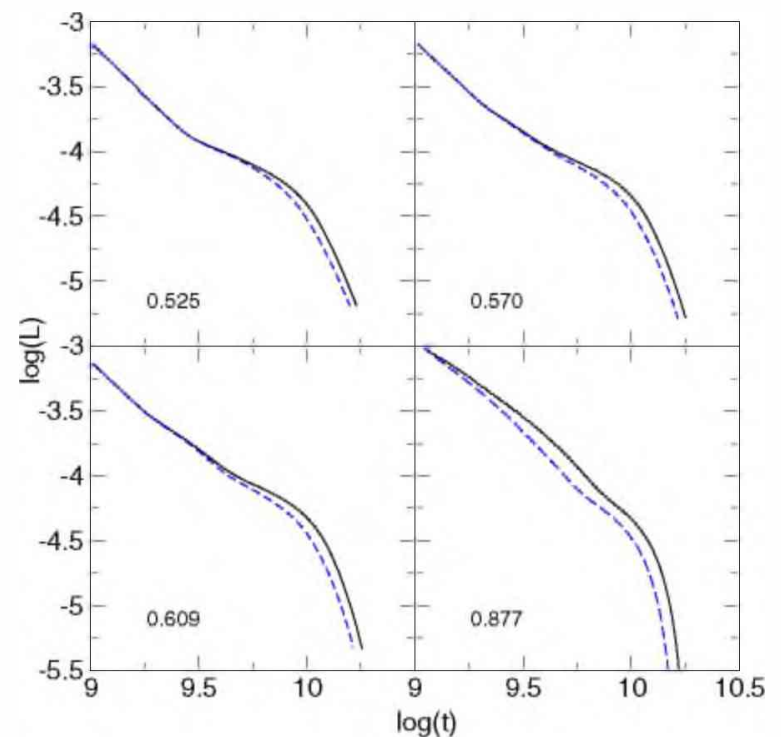

Figure 7. Cooling curves at advanced stages in the white dwarf evolution for our sequences of masses $0.525 M_{\odot}$ (upper left panel), $0.570 M_{\odot}$ (upper right panel), $0.609 M_{\odot}$ (bottom left panel), and $0.877 M_{\odot}$ (bottom right panel). Solid lines correspond to the case in which both latent heat and carbon-oxygen phase separation are considered, while dashed lines correspond to the situation when carbon-oxygen phase separation is neglected. The metallicity of progenitor stars is $Z=0.01$.

(A color version of this figure is available in the online journal.)

For the sake of comparison, in the bottom left panel of this figure we also show as a dashed-dotted line the profile obtained by Salaris et al. (1997) for a white dwarf of $0.61 M_{\odot}$, a mass value very close to that of this panel. Note that the central oxygen abundance in the $0.61 M_{\odot}$ white dwarf of Salaris et al. (1997) is somewhat higher than that of our $0.609 M_{\odot}$ white dwarf. This is mostly because we use the value of the NACRE compilation for the ${ }^{12} \mathrm{C}(\alpha, \gamma){ }^{16} \mathrm{O}$ reaction rate (Angulo et al. 1999), which is smaller than the rate of Caughlan et al. (1985) adopted by Salaris et al. (1997). However, another point that has to be considered in this comparison is the fact that the white dwarf mass in Salaris et al. (1997) is assessed at the first thermal pulse. Thus, for a given white dwarf mass, our progenitor stars are less massive than those of Salaris et al. (1997), see our Figure 2. Because of this effect alone, our white dwarf model should be characterized 


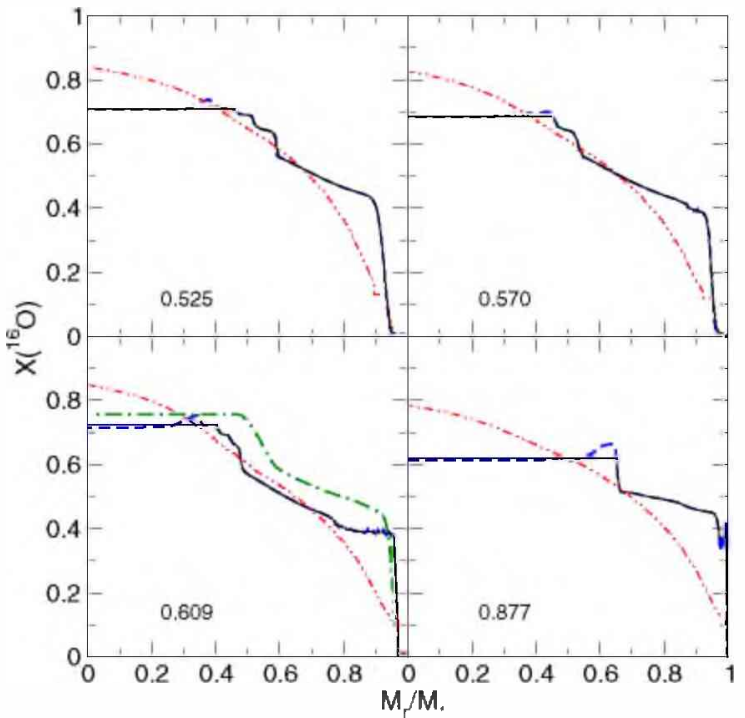

Figure 8. Oxygen abundance (by mass) profiles for four selected white dwarfs sequences with stellar masses (in solar masses) as labeled in each panel. Dashed lines show the abundance distribution shortly after the AGB stage. Solid lines show the chemical profile after Rayleigh-Taylor rehomogenization has occurred in the core and dot-dot-dashed lines show the profiles after carbon-oxygen phase separation upon crystallization has finished. For comparison, in the bottom left panel we also show with a dash-dotted line the oxygen profile of Salaris et al. (1997) after the rehomogenization phase of a white dwarf with mass $0.609 \mathrm{M}_{\odot}$. Note that in all cases, the initial chemical distribution in the core has been markedly altered by the crystallization process. The metallicity of the progenitor stars is $Z=0.01$.

(A color version of this figure is available in the online journal.)

by a higher central oxygen abundance than would have resulted from a more massive progenitor-see Althaus et al. (2010). Thus, on one hand we expect a lower oxygen abundance in our model because of our adopted cross section for the ${ }^{12} \mathrm{C}(\alpha, \gamma){ }^{16} \mathrm{O}$ reaction rate, but on the other hand, we expect a higher oxygen abundance because of the lower initial mass of our progenitor star. The net effect is that the central oxygen abundance in our model results to be somewhat lower than that in the Salaris et al. (1997) model. Finally, the treatment of extra-mixing episodes during core helium-burning, which are well known to influence the final carbon-oxygen stratification, leads to some differences in the expected composition. In our simulation, extra-mixing episodes are treated as diffusion processes (Herwig et al. 1997), while in Salaris et al. (1997) extra-mixing is considered as a semiconvective process. However, as shown by Straniero et al. (2003) both treatments give rise to a quite similar core chemical stratification, and thus no appreciable difference in the central oxygen abundance should be expected from these treatments.

\subsection{Colors and the Blue Hook}

The molecular hydrogen formation at low effective temperatures also affects the evolution of our models in the color-magnitude diagram, as shown in Figure 9, which displays the run of the absolute visual magnitude $M_{V}$ in terms of four standard colors: $V-I, U-V, B-V$, and $V-R$. For the evolutionary stages computed in this work the turn to the blue at $M_{V} \approx 17$ is noticeable for the $V-I$ and $V-R$ colors. This effect is due to the $\mathrm{H}_{2}-\mathrm{H}_{2}$ collision-induced absorption over the infrared spectral regions, which forces the stellar flux to emerge at shorter wavelengths. Note that in this diagram, all our sequences are expected to become markedly blue when the $V-I$ and $V-R$ color indices are used. For instance, for the $V-I$
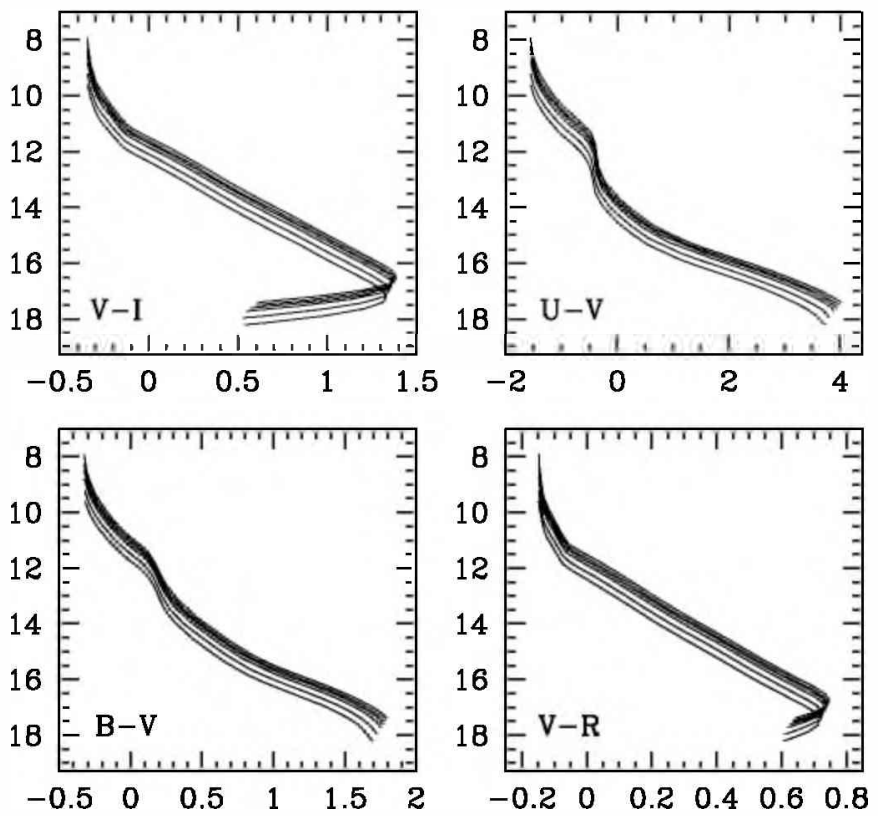

Figure 9. Absolute visual magnitude $M_{V}$ in terms of the color index $V-I$ (upper left panel), $U-V$ (upper right panel), $B-V$ (bottom left panel), and $V-R$ (bottom right panel), for the complete evolutionary tracks of our sequences with $Z=0.01$, from top to bottom: $0.525 M_{\odot}, 0.570 M_{\odot}, 0.609 M_{\odot}, 0.659 M_{\odot}$, $0.767 M_{\odot}$, and $0.878 M_{\odot}$

color index, the turnoff point occurs between $M_{V}=16.5$ and 17.2 , depending on the stellar mass value. This range corresponds to luminosities between $\log \left(L / L_{\odot}\right)=-4.6$ and -5.0 , and cooling ages between 11.9 and $15 \mathrm{Gyr}$ for our sequences with carbon-oxygen phase separation. On the other hand, $U-V$ and $B-V$ are sensitive to $\mathrm{Ly} \alpha$ broadening by $\mathrm{H}-\mathrm{H}_{2}$ collisions. This reduces the emergent flux in the ultraviolet and blue regions, thus producing redder color indices for the models cooler than about $T_{\mathrm{eff}}=5000 \mathrm{~K}(U-V>1.0$ and $B-V>0.8)$.

\section{CONCLUSIONS}

We have computed a set of cooling sequences for hydrogenrich white dwarfs, which are appropriate for precision white dwarf cosmochronology. Our evolutionary sequences have been self-consistently evolved from ZAMS, through the core hydrogen- and helium-burning evolutionary phases to the thermally pulsing AGB and, ultimately, to the white dwarf stage. This has been done for white dwarf progenitors with two different metallicities. For the first set of evolutionary sequences, we have adopted solar metallicity. This allows us to obtain accurate ages for white dwarfs in the local Galactic disk. The second set of cooling sequences corresponds to a metallicity typical of most Galactic globular clusters, $Z=0.001$, thus allowing to obtain accurate ages for metal-poor stellar systems. To the best of our knowledge, this is the first set of self-consistent evolutionary sequences covering different initial masses and metallicities.

Our main findings can be summarized as follows. First, our evolutionary sequences correctly reproduce the observed initialto-final mass relationship of white dwarfs. Specifically, our evolutionary calculations are in excellent agreement with the recent results of Salaris et al. (2009) for white dwarfs with solarmetallicity progenitors. Second, we corroborate the importance of convective coupling at low luminosity in the cooling of white dwarfs, as originally suggested by Fontaine et al. (2001). Third, we demonstrate the importance of residual hydrogen-burning 
in white dwarfs resulting from low-metallicity progenitors. Fourth, we also confirm the importance of carbon-oxygen phase separation upon crystallization in good qualitative agreement with the results of García-Berro et al. (1988a, 1988b), Segretain et al. (1994), and Salaris et al. (1997, 2000). Although the computed delays are smaller than those previously estimated by Segretain et al. (1994), they are larger than those computed by Salaris et al. (2000), and are by no means negligible if precision white dwarf cosmochronology is to be done. However, we would like to mention that these delays depend crucially on the previous evolutionary history of white dwarf progenitors and, particularly, on the rate of the ${ }^{12} \mathrm{C}(\alpha, \gamma){ }^{16} \mathrm{O}$ nuclear reaction, as well as on the adopted treatment for convective mixing. Additionally, since our evolutionary sequences rely on state-ofthe-art non-gray model atmospheres, they reproduce the wellknown blue hook of very old hydrogen-rich white dwarfs caused by $\mathrm{H}_{2}-\mathrm{H}_{2}$ collision-induced absorption (Hansen 1999). Finally, we show the impact of $\mathrm{Ly} \alpha$ quasi-molecular opacity on the evolution of cool white dwarfs in the color-magnitude diagram.

We would like to emphasize that our full treatment of the entire evolutionary history of white dwarfs has allowed us to obtain consistent white dwarf initial configurations. In particular, the mass of the hydrogen-rich envelope and of the helium buffer were obtained from evolutionary calculations, instead of using typical values and artificial initial white dwarf models. This has implications for the cooling rates of old white dwarfs, as the thicknesses of these outer layers control the cooling speed of such white dwarfs. Another important issue which we would like to mention is that our calculations also yield self-consistent interior chemical profiles. This also has relevance for the cooling of white dwarfs, as the release of latent heat and gravitational energy due to carbon-oxygen phase separation upon crystallization crucially depend on the previous evolutionary history of white dwarfs. Also, the chemical stratification of white dwarf progenitors is important for correctly computing the specific heat of white dwarf interiors. We would also like to stress that the evolutionary tracks of cooling white dwarfs presented here have been computed with the most accurate physical inputs and with a high degree of detail and realism. In particular, our calculations include nuclear burning at the very early phases of white dwarf evolution - which is important to determine the final thickness of the hydrogen-rich envelope-diffusion and gravitational settling - which are important to shape the profiles of the outer layers-accurate neutrino emission rates-which control the cooling at high luminosities-crystallization and phase separation of carbon and oxygen - which dominate the cooling times at low luminosities-a very detailed equation of state-which is important in all the evolutionary phases-and improved non-gray model atmospheres - which allow for a precise determination of white dwarf colors and outer boundary conditions for the evolving models. Finally, we would like to remark that these evolutionary sequences are also important for the calculation of self-consistent models of pulsating DA white dwarfs. In summary, we consider that our cooling sequences represent a landmark in the calculation of white dwarf evolutionary tracks, and they open a new era to precision white dwarf cosmochronology.

This research was supported by AGENCIA: Programa de Modernización Tecnológica BID 1728/OC-AR, by the AGAUR, by MCINN grant AYA2008-04211-C02-01, by the European Union FEDER funds and by PIP 112-200801-00940 grant from CONICET. L.G.A. also acknowledges a PIV grant of the AGAUR of the Generalitat de Catalunya. I.R. also acknowledges an FPU grant of the Spanish MEC.

\section{REFERENCES}

Alexander, D. R., \& Ferguson, J. W. 1994, ApJ, 437, 879

Althaus, L. G., Córsico, A. H., Bischoff-Kim, A., Romero, A. D., Renedo, I., García-Berro. E.. \& Miller Bertolami, M. M. 2010, ApJ, in press (arXiv:1005.2612)

Althaus, L. G., Córsico, A. H., Torres, S., \& García-Berro, E. 2009a, A\&A. 494, 1021

Althaus. L. G., García-Berro. E.. Córsico, A. H.. Miller Bertolami. M. M.. \& Romero, A. D. 2009b, ApJ, 693, L23

Althaus, L. G., García-Berro, E., Isern, J., Córsico, A. H., \& Rohrmann, R. D 2007, A\&A, 465, 249

Althaus, L. G., Miller Bertolami. M. M., Córsico, A. H.. García-Berro. E.. \& Gil-Pons, P. 2005a, A\&A, 440, L1

Althaus, L. G., Panei, J. A., Miller Bertolami, M. M., García-Berro, E., Córsico, A. H., Romero, A. D., Kepler, S. O., \& Rohrmann, R. D. 2009c. ApJ, 704. 1605

Althaus, L. G., Panei, J. A., Romero, A. D., Rohrmann, R. D., Córsico, A. H. García-Berro, E., \& Miller Bertolami, M. M. 2009d, A\&A, 502, 207

Althaus, L. G., Serenelli, A. M., Córsico, A. H., \& Montgomery, M. H. 2003. A\&A. 404, 593

Althaus, L. G., Serenelli, A. M., Panei, J. A., Córsico, A. H., García-Berro, E., \& Scóccola, C. G. 2005b, A\&A, 435, 631

Angulo, C., et al. 1999, Nucl. Phys. A, 656, 3

Barrado y Navascués, D., Stauffer, J. R.. Bouvier. J., \& Martín. E. L. 2001. ApJ. 546,1006

Benvenuto, O. G., \& Althaus, L. G. 1999, MNRAS, 303, 30

Benvenuto. O. G., García-Berro. E., \& Isern. J. 2004. Phys. Rev. D. 69. 082002

Bergeron, P., Ruiz, M. T., \& Leggett, S. K. 1997, ApJS, 108, 339

Burgers, J. M. 1969, Flow Equations for Composite Gases (New York: Academic)

Casagrande, L.. Flynn. C.. Portinari. L., Girardi, L., \& Jimenez, R. 2007. MNRAS, 382, 1516

Cassisi, S., Potekhin, A. Y., Pietrinferni, A., Catelan, M., \& Salaris, M. 2007, ApJ, 661, 1094

Catalán. S.. Isern. J., García-Berro, E., \& Ribas, I. 2008a. MNRAS, 387. 1693

Catalán, S., Isern, J., García-Berro, E., Ribas, I., Allende Prieto, C.. \& Bonanos. A. Z. 2008b, A\&A, 477, 213

Caughlan. G. R.. Fowler. W. A.. Harris. M. J.. \& Zimmermann. B. A. 1985. At. Data Nucl. Data Tables, 32. 197

Córsico, A. H., Benvenuto, O. G., Althaus, L. G., Isern, J., \& García-Berro, E. 2001, New Astron., 6, 197

Díaz-Pinto. A.. García-Berro, E.. Hernanz. M.. Isern. J., \& Mochkovitch. R. 1994, A\&A, 282, 86

Domínguez, I., Chieffi, A., Limongi. M., \& Straniero, O. 1999, ApJ, 524, 226

Fontaine, G.. \& Brassard. P. 2008. PASP. 120. 1043

Fontaine, G., Brassard. P., \& Bergeron. P. 2001, PASP. 113, 409

Flynn, C. 2004, PASA, 21, 126

García-Berro, E., Hernanz, M., Isern, J., \& Mochkovitch, R. 1988a, Nature, 333. 642

García-Berro, E., Hernanz, M., Isern, J., \& Mochkovitch, R. 1995, MNRAS. 277,801

García-Berro, E., Hernanz, M., Mochkovitch, R., \& Isern, J. 1988b, A\&A. 193. 141

García-Berro, E., Torres, S., Isern, J., \& Burkert, A. 1999. MNRAS, 302, 173

Grevesse, N., \& Sauval, A. J. 1998, Space Sci. Rev., 85, 161

Haft. M., Raffelt. G.. \& Weiss, A. 1994. ApJ. 425. 222

Hansen, B. M. S. 1998, Nature, 394, 860

Hansen. B. M. S. 1999, ApJ, 520, 680

Hansen. B. M. S.. \& Liebert. J. 2003. ARA\&A. 41. 465

Hansen. B. M. S., et al. 2002, ApJ, 574, L155

Hansen, B. M. S., et al. 2007, ApJ, 671, 380

Herwig. F., Blocker. T.. Schonberner. D.. \& El Eid. M. 1997. A\&A. 324, L81

Iben. I., Jr.. \& MacDonald, J. 1985. ApJ. 296. 540

Iben, I., Jr., \& MacDonald, J. 1986, ApJ, 301, 164

Iglesias, C. A., \& Rogers, F. J. 1996, ApJ, 464, 943

Isern. J.. García-Berro. E.. Hernanz. M.. \& Chabrier. G. 2000. ApJ. 528. 397

Isern, J., García-Berro, E., Hernanz, M., Mochkovitch, R., \& Torres, S. 1998, ApJ, 03, 239

Isern, J., García-Berro, E., Torres, S., \& Catalán, S. 2008, ApJ, 682, L109

Isern. J., Hernanz, M., \& Garcia-Berro, E. 1992, ApJ, 392, L23

Isern, J., Mochkovitch, R., Garcia-Berro, E., \& Hernanz, M. 1997, ApJ, 485. 308 
Itoh, N.. Hayashi, H., Nishikawa. A.. \& Kohyama. Y. 1996, ApJS, 102, 411 Kalirai, J. S., Hansen, B. M. S., Kelson. D. D., Reitzel, D. B., Rich. R. M., \& Richer, H. B. 2008, ApJ, 676, 594

Kalirai, J. S., Ventura, P., Richer, H. B., Fahlman. G. G., Durrell, P. R., D’Antona, F., \& Marconi, G. 2001, AJ, 122, 3239

Kołos. W.. \& Wolniewicz. L. 1965. J. Chem. Phys.. 43. 2429

Kowalski. P. M.. \& Saumon. D. 2006. ApJ, 651, L137

Kulander, K. C., \& Guest, M. F. 1979. J. Phys. B: At. Mol. Phys., 12, L501

Lugaro, M., Herwig, F., Lattanzio, J. C.. Gallino, R., \& Straniero, O. 2003, ApJ, 586, 1305

Magni. G.. \& Mazzitelli, I. 1979. A\&A. 72. 134

Marigo, P. 2002, A\&A, 387, 507

Marigo, P., \& Aringer, B. 2009, A\&A, 508, 1539

Mestel, L. 1952, MNRAS, 112, 583

Miller Bertolami, M. M., \& Althaus, L. G. 2006, A\&A. 454, 845

Miller Bertolami, M. M., Althaus, L. G.. Unglaub, K., \& Weiss, A. 2008, A\&A. 491,253

Petsalakis, I. D., Theodorakopoulos, G., Wright, J. S., \& Hamilton, I. P. 1988. J. Chem. Phys., 88, 7633

Prada Moroni. P. G.. \& Straniero, O. 2002. ApJ, 581. 585

Prada Moroni, P. G., \& Straniero, O. 2007. A\&A. 466, 1043

Roach. A. C., \& Kuntz, P. J. 1986, J. Chem. Phys., 84, 822

Rohrmann, R. D., Serenelli, A. M., Althaus, L. G., \& Benvenuto, O. G. 2002. MNRAS. 335, 499
Salaris, M., Domínguez, I.. García-Berro, E.. Hernanz, M.. Isern, J., \& Mochkovitch, R. 1997. ApJ, 486, 413

Salaris, M., García-Berro, E., Hernanz, M., Isern, J., \& Saumon. D. 2000, ApJ. 544,1036

Salaris, M., Serenelli, A., Weiss, A., \& Miller Bertolami, M. 2009, ApJ, 692. 1013

Schroder, K.-P., \& Cuntz, M. 2005, ApJ, 630, L73

Segretain, L., \& Chabrier, G. 1993, A\&A, 271, L13

Segretain, L., Chabrier, G., Hernanz, M., Garcia-Berro, E., Isern, J., \& Mochkovitch, R. 1994. ApJ, 434, 641

Straniero, O., Domínguez. I.. Imbriani. G., \& Piersanti. L. 2003. ApJ. 583. 878

Tassoul, M., Fontaine, G., \& Winget, D. E. 1990, ApJS, 72, 335

Torres, S., García-Berro, E., Burkert, A., \& Isern, J. 2002. MNRAS, 336, 971

Unglaub, K., \& Bues, I. 2000, A\&A, 359, 1042

Vassiliadis, E., \& Wood. P. R. 1993, ApJ, 413, 641

Weiss, A., \& Ferguson, J. W. 2009, A\&A, 508, 1343

Winget, D. E., Hansen, C. J., Liebert, J., van Horn, H. M., Fontaine, G., Nather. R. E., Kepler. S. O.. \& Lamb. D. Q. 1987, ApJ, 315, L77

Winget. D. E.. \& Kepler. S. O. 2008. ARA\&A. 46, 157

Wood, M. A. 1992, ApJ, 386, 539

Wood, M. A. 1995, in Proc. 9th European Workshop on White Dwarfs, ed. D. Koester \& K. Werner (Lecture Notes in Physics, Vol. 443; Berlin: Springer) 41 